\title{
Mechanical Properties of Single-walled Carbon Nanotubes Simulated with AIREBO Force-Field
}

\author{
Michał Białoskórski ${ }^{1,2}$, Jarosław Rybicki ${ }^{1,2,3}$ \\ ${ }^{1}$ Gdansk University of Technology, Faculty of Applied Physics and Mathematics \\ ul. Narutowicza 11/12, 80-233 Gdansk, Poland \\ ${ }^{2}$ TASK Computer Center, \\ ul. Narutowicza 11/12, 80-233 Gdansk, Poland \\ ${ }^{3}$ Koszalin University of Technology, Institute of Nanotechnology, Mechatronics and Vacuum Techniques \\ ul. Śniadeckich, Koszalin, Poland \\ e-mail:m.bialoskorski@task.gda.pl; ryba@pg.gda.pl
}

(Received: 20 June 2012; accepted: 27 June 2012; published online: 14 August 2012)

\begin{abstract}
In this work we determined the mechanical properties (Young's modulus, Poisson's ratio, and shear modulus) of 400 single-walled carbon nanotubes of radii from 2.1; $((0,5)$ nanotube) to $17.3 \AA((0,45)$ nanotube $)$. All nanotubes were simulated with AIREBO forcefield. It turns out that zigzag nanotubes are mechanically more resistant than armchair nanotubes.
\end{abstract}

Key words: carbon nanotubes, mechanical properties, molecular dynamics, AIREBO

Single-walled carbon nanotubes (SWCNT) have been the subject of research for years. Due to the unique mechanical and electrical characteristics they continue to be a promising material for future practical applications [1]. Most of the researchers' efforts have been focused on the study of the electrical properties of SWCNTs, while the mechanical properties have been studied much less frequently. The experimental works have been related to single nanotube types for which the defined values of individual elastomechanical constants are given or the mechanical properties of mixtures of nanotubes of different types are characterized in very general terms [2-4]. This brief paper presents the results of systematic studies of the mechanical properties of nanotubes with all chiralities starting from $(5,0)$ to $(20,20)$ and additionally $(0,25)$, $(0,30),(0,35),(0,40),(0,45)$ and $(25,25)$, corresponding to the diameters of $4.2 \AA$ to $34.6 \AA$, respectively. All the studied tubes were about $170 \AA$ long.

The samples were studied in a series of computer simulations. The simulations were performed with the molecular dynamics method using the AIREBO potential
[5] and a Nosé-Hoover thermostat [6], as implemented in the nanoMD program [7]. The calculations were performed at a constant temperature of $300 \mathrm{~K}$. The simulations consisted in longitudinal stretching and compressing of samples at a constant relative velocity of the nanotube ends of $0.001 \mathrm{fs} / \AA$. All the nanotubes were relaxed before application of external interactions within 100000 steps with 0.5 fs timestep.

The value of Young's modulus, $Y$, was obtained from the stress dependence, $\sigma=F / S(F-$ longitudinal force measured at the ends of the sample, $S$ - nanotube crosssection, calculated as the nanotube circumference multiplied by the nanotube wall thickness equal to $3.4 \AA$, as is common used) on the longitudinal strain $\varepsilon$, according to the formula:

$$
Y=\frac{\sigma}{\varepsilon}
$$

Poisson's ratio, $v$, was determined from the sample radial strain dependence, $\varepsilon_{r}$, on the longitudinal strain, $\varepsilon$, according to the formula: 


$$
v=\frac{\varepsilon_{r}}{\varepsilon} .
$$

The values $Y$ and $v$ were determined in two ways: a) assuming the linear dependence, $\sigma(\varepsilon)$ and $v(\varepsilon)$, for strains $|\varepsilon|<0.015$, b) assuming the linear dependence separately in the ranges of $-0.02<\varepsilon<0$ (for compressing - subscript $s$ in symbols) and $0>\varepsilon>0.04$ (for stretching - subscript $r$ ), thus reflecting the minor nonlinearity of $\sigma(\varepsilon)$ and $v(\varepsilon)$.

As far as the obtained results are considered special attention should be paid to the dependence of the mechanical properties on the nanotube chirality. When determining the parameters from the strain range symmetric with respect to zero ( $Y$ and $v$ values in the Table), the values do not show any dependence on the chirality. If the mechanical parameters are determined unilaterally, i.e. separately for compressing and stretching, the values $Y$ and $v$ are dependent on the nanotube chirality $\left(Y_{s}, Y_{r}, v_{\mathrm{s}}\right.$ and $v_{\mathrm{r}}$ in the Table where the relationships of $Y_{s}, Y_{r}$ and $v_{s} / v_{r}$ are also given). For zigzag nanotubes (chiralities $(0, n))$ Young's modulus for stretching is greater than Young's modulus for armchair nanotubes (chiralities of $(n, n))$, and vice versa - for compression - Young's modulus of zigzag nanotubes is smaller than the modulus for armchair nanotubes.

Simulations of torsional vibrations around the nanotube axis had to be performed to calculate the shear modulus, $G$. When setting the frequency of torsional vibrations the shear modulus can be defined as:

$$
G=16 L^{2} f^{2} \rho
$$

\begin{tabular}{|c|c|c|c|c|c|c|c|c|c|c|c|}
\hline Chirality & $R_{0}$ & $L_{0}$ & $Y$ & $Y_{s}$ & $Y_{r}$ & $v$ & $v_{s}$ & $v_{r}$ & $G$ & $Y_{\mathrm{s}} / Y_{r}$ & $v_{S} / v_{r}$ \\
\hline 1 & 2 & 3 & 4 & 5 & 6 & 7 & 8 & 9 & 10 & 11 & 12 \\
\hline$(5,0)$ & 2.1 & 162 & 0.91 & 0.90 & 0.81 & 0.08 & 0.10 & 0.04 & & 1.11 & 2.45 \\
\hline$(0,5)$ & 2.1 & 164.4 & 0.97 & 1.07 & 0.82 & 0.08 & 0.12 & 0.05 & & 1.31 & 2.49 \\
\hline$(6,0)$ & 2.4 & 165.1 & 0.88 & 0.92 & 0.79 & 0.10 & 0.12 & 0.05 & 6.03 & 1.17 & 2.27 \\
\hline$(0,6)$ & 2.4 & 165.7 & 0.93 & 0.96 & 0.81 & 0.10 & 0.12 & 0.06 & & 1.18 & 2.01 \\
\hline$(0,7)$ & 2.8 & 168.3 & 0.92 & 0.89 & 0.80 & 0.11 & 0.15 & 0.07 & & 1.11 & 2.22 \\
\hline$(0,8)$ & 3.2 & 168.6 & 0.91 & 0.92 & 0.81 & 0.14 & 0.17 & 0.07 & & 1.13 & 2.29 \\
\hline$(8,0)$ & 3.2 & 168 & 0.87 & 0.95 & 0.82 & 0.13 & 0.17 & 0.08 & 6.05 & 1.16 & 2.19 \\
\hline$(0,9)$ & 3.5 & 168.8 & 0.86 & 0.89 & 0.82 & 0.14 & 0.18 & 0.08 & & 1.08 & 2.19 \\
\hline$(0,10)$ & 3.9 & 168.9 & 0.87 & 0.91 & 0.83 & 0.15 & 0.20 & 0.09 & & 1.10 & 2.30 \\
\hline$(11,0)$ & 4.3 & 168.3 & 0.85 & 0.90 & 0.81 & 0.17 & 0.21 & 0.09 & 5.88 & 1.10 & 2.39 \\
\hline$(0,11)$ & 4.3 & 169 & 0.86 & 0.90 & 0.82 & 0.16 & 0.21 & 0.09 & & 1.10 & 2.40 \\
\hline$(0,12)$ & 4.7 & 169 & 0.86 & 0.87 & 0.82 & 0.17 & 0.22 & 0.09 & & 1.06 & 2.37 \\
\hline$(12,0)$ & 4.7 & 168.3 & 0.84 & 0.85 & 0.82 & 0.17 & 0.21 & 0.09 & 5.82 & 1.03 & 2.19 \\
\hline$(0,13)$ & 5.1 & 169 & 0.85 & 0.88 & 0.82 & 0.18 & 0.22 & 0.09 & & 1.07 & 2.36 \\
\hline$(13,0)$ & 5.1 & 168.3 & 0.87 & 0.89 & 0.82 & 0.18 & 0.23 & 0.10 & 5.78 & 1.09 & 2.37 \\
\hline$(14,0)$ & 5.4 & 168.3 & 0.89 & 0.89 & 0.82 & 0.18 & 0.23 & 0.10 & 5.73 & 1.09 & 2.33 \\
\hline$(0,14)$ & 5.4 & 169 & 0.90 & 0.90 & 0.82 & 0.18 & 0.23 & 0.10 & & 1.09 & 2.24 \\
\hline$(0,15)$ & 5.8 & 169 & 0.90 & 0.92 & 0.83 & 0.19 & 0.23 & 0.10 & & 1.11 & 2.26 \\
\hline$(15,0)$ & 5.8 & 168.3 & 0.92 & 0.91 & 0.82 & 0.18 & 0.23 & 0.10 & 5.63 & 1.11 & 2.28 \\
\hline$(16,0)$ & 6.2 & 168.4 & 0.85 & 0.88 & 0.83 & 0.19 & 0.24 & 0.10 & 5.74 & 1.07 & 2.46 \\
\hline$(0,16)$ & 6.2 & 169 & 0.90 & 0.90 & 0.82 & 0.19 & 0.24 & 0.10 & & 1.10 & 2.31 \\
\hline$(17,0)$ & 6.6 & 168.4 & 0.82 & 0.87 & 0.82 & 0.18 & 0.24 & 0.10 & 5.93 & 1.06 & 2.36 \\
\hline$(0,17)$ & 6.6 & 171.1 & 0.85 & 0.85 & 0.82 & 0.19 & 0.23 & 0.10 & & 1.03 & 2.29 \\
\hline$(18,0)$ & 7 & 168.4 & 0.83 & 0.85 & 0.82 & 0.19 & 0.25 & 0.10 & 5.96 & 1.04 & 2.43 \\
\hline$(0,18)$ & 7 & 171.2 & 0.83 & 0.85 & 0.84 & 0.19 & 0.22 & 0.11 & & 1.02 & 2.12 \\
\hline$(0,19)$ & 7.3 & 171.2 & 0.83 & 0.85 & 0.83 & 0.19 & 0.24 & 0.10 & & 1.02 & 2.34 \\
\hline$(19,0)$ & 7.3 & 168.4 & 0.83 & 0.83 & 0.82 & 0.19 & 0.25 & 0.11 & 5.93 & 1.02 & 2.29 \\
\hline
\end{tabular}

Table 1. Mechanical properties of SWCNTs. The values of $R_{0}, L_{0}$ are given in $\AA . Y, Y_{s}, Y_{r}$ are in TPa and $G$ is in GPa 


\begin{tabular}{|c|c|c|c|c|c|c|c|c|c|c|c|}
\hline 1 & 2 & 3 & 4 & 5 & 6 & 7 & 8 & 9 & 10 & 11 & 12 \\
\hline$(0,20)$ & 7.7 & 171.2 & 0.82 & 0.88 & 0.83 & 0.20 & 0.24 & 0.10 & & 1.06 & 2.26 \\
\hline$(25,0)$ & 9.6 & 168.4 & 0.83 & 0.84 & 0.82 & 0.21 & 0.25 & 0.11 & & 1.02 & 2.34 \\
\hline$(30,0)$ & 12 & 168.3 & 0.86 & 0.85 & 0.82 & 0.22 & 0.24 & 0.11 & & 1.03 & 2.25 \\
\hline$(35,0)$ & 13 & 168.3 & 0.85 & 0.81 & 0.82 & 0.21 & 0.25 & 0.11 & & 0.99 & 2.28 \\
\hline$(40,0)$ & 15 & 168.3 & 0.82 & 0.81 & 0.82 & 0.23 & 0.23 & 0.11 & & 0.99 & 2.11 \\
\hline$(45,0)$ & 17 & 168.3 & 0.85 & 0.85 & 0.82 & 0.22 & 0.23 & 0.11 & & 1.03 & 2.10 \\
\hline$(3,3)$ & 2.1 & 168.7 & 0.86 & 0.92 & 0.70 & 0.12 & 0.11 & 0.17 & & 1.31 & 0.68 \\
\hline$(4,4)$ & 2.7 & 169.5 & 0.88 & 0.92 & 0.71 & 0.17 & 0.14 & 0.20 & 6.93 & 1.29 & 0.68 \\
\hline$(5,5)$ & 3.4 & 170.3 & 0.87 & 0.94 & 0.72 & 0.19 & 0.16 & 0.22 & 6.74 & 1.30 & 0.73 \\
\hline$(6,6)$ & 4 & 170.1 & 0.88 & 0.94 & 0.71 & 0.20 & 0.17 & 0.22 & 6.58 & 1.32 & 0.79 \\
\hline$(7,7)$ & 4.7 & 170 & 0.81 & 0.97 & 0.74 & 0.20 & 0.17 & 0.23 & 6.48 & 1.30 & 0.74 \\
\hline$(8,8)$ & 5.4 & 171.1 & 0.82 & 0.96 & 0.73 & 0.21 & 0.18 & 0.23 & 6.35 & 1.30 & 0.76 \\
\hline$(9,9)$ & 6 & 171 & 0.83 & 1.00 & 0.73 & 0.20 & 0.18 & 0.23 & 6.41 & 1.36 & 0.75 \\
\hline$(10,10)$ & 6.7 & 170.9 & 0.83 & 0.93 & 0.73 & 0.21 & 0.17 & 0.23 & 6.44 & 1.27 & 0.74 \\
\hline$(11,11)$ & 7.4 & 170.9 & 0.87 & 0.94 & 0.74 & 0.21 & 0.18 & 0.23 & 6.49 & 1.27 & 0.77 \\
\hline$(12,12)$ & 8 & 170.8 & 0.85 & 0.92 & 0.73 & 0.21 & 0.18 & 0.23 & 6.37 & 1.26 & 0.77 \\
\hline$(13,13)$ & 8.7 & 170.8 & 0.88 & 0.94 & 0.73 & 0.21 & 0.19 & 0.23 & 6.53 & 1.29 & 0.80 \\
\hline$(14,14)$ & 9.4 & 170.8 & 0.86 & 0.94 & 0.74 & 0.21 & 0.18 & 0.23 & 6.45 & 1.26 & 0.75 \\
\hline$(15,15)$ & 10 & 170.8 & 0.85 & 0.93 & 0.74 & 0.21 & 0.18 & 0.23 & 6.50 & 1.26 & 0.75 \\
\hline$(16,16)$ & 11 & 170.8 & 0.86 & 0.93 & 0.74 & 0.21 & 0.18 & 0.24 & 6.51 & 1.26 & 0.74 \\
\hline$(17,17)$ & 11 & 170.7 & 0.86 & 0.93 & 0.74 & 0.21 & 0.16 & 0.23 & 6.40 & 1.26 & 0.72 \\
\hline$(18,18)$ & 12 & 170.7 & 0.87 & 0.93 & 0.73 & 0.21 & 0.18 & 0.23 & 6.39 & 1.26 & 0.76 \\
\hline$(19,19)$ & 13 & 170.7 & 0.78 & 0.94 & 0.74 & 0.17 & 0.17 & 0.23 & 6.54 & 1.28 & 0.75 \\
\hline$(20,20)$ & 13 & 170.7 & 0.87 & 0.94 & 0.73 & 0.21 & 0.15 & 0.23 & 6.51 & 1.28 & 0.65 \\
\hline$(25,25)$ & 17 & 170.7 & 0.84 & 0.95 & 0.73 & 0.22 & 0.16 & 0.23 & & 1.29 & 0.71 \\
\hline$(2,3)$ & 1.8 & 166.3 & 0.87 & 0.93 & 0.74 & 0.09 & 0.16 & 0.07 & & 1.26 & 2.15 \\
\hline$(3,2)$ & 1.8 & 166.4 & 0.89 & 0.89 & 0.70 & 0.12 & 0.11 & 0.10 & & 1.27 & 1.14 \\
\hline$(1,4)$ & 1.9 & 164.2 & 0.92 & 0.96 & 0.79 & 0.06 & 0.20 & 0.02 & & 1.22 & 11.36 \\
\hline$(4,1)$ & 1.9 & 163.7 & 0.91 & 0.90 & 0.79 & 0.08 & 0.03 & 0.02 & & 1.14 & 1.35 \\
\hline$(4,2)$ & 2.1 & 167.2 & 0.86 & 0.96 & 0.74 & 0.09 & 0.07 & 0.10 & & 1.30 & 0.72 \\
\hline$(2,4)$ & 2.1 & 167.5 & 0.93 & 0.95 & 0.75 & 0.09 & 0.08 & 0.10 & & 1.27 & 0.81 \\
\hline$(1,5)$ & 2.2 & 166.8 & 0.90 & 0.94 & 0.79 & 0.08 & 0.10 & 0.04 & & 1.19 & 2.48 \\
\hline$(5,1)$ & 2.2 & 166.2 & 0.88 & 0.91 & 0.80 & 0.06 & 0.08 & 0.05 & & 1.15 & 1.65 \\
\hline$(3,4)$ & 2.4 & 169.7 & 0.88 & 0.91 & 0.71 & 0.12 & 0.06 & 0.16 & & 1.29 & 0.35 \\
\hline$(2,5)$ & 2.5 & 168.8 & 0.87 & 0.94 & 0.76 & 0.10 & 0.10 & 0.09 & & 1.24 & 1.07 \\
\hline$(5,2)$ & 2.5 & 168.4 & 0.92 & 0.99 & 0.77 & 0.10 & 0.11 & 0.09 & & 1.29 & 1.22 \\
\hline$(1,6)$ & 2.6 & 168.3 & 0.93 & 0.97 & 0.81 & 0.09 & 0.14 & 0.05 & & 1.20 & 2.95 \\
\hline$(6,1)$ & 2.6 & 167.4 & 0.91 & 0.91 & 0.79 & 0.10 & 0.12 & 0.05 & 6.34 & 1.15 & 2.24 \\
\hline$(5,3)$ & 2.8 & 169.7 & 0.84 & 0.94 & 0.72 & 0.14 & 0.13 & 0.15 & 7.02 & 1.29 & 0.88 \\
\hline$(3,5)$ & 2.8 & 169.9 & 0.87 & 0.91 & 0.72 & 0.13 & 0.12 & 0.14 & & 1.26 & 0.83 \\
\hline$(2,6)$ & 2.9 & 169.4 & 0.92 & 0.95 & 0.79 & 0.12 & 0.13 & 0.10 & & 1.20 & 1.32 \\
\hline$(6,2)$ & 2.9 & 168.6 & 0.89 & 0.97 & 0.79 & 0.12 & 0.13 & 0.10 & 6.56 & 1.23 & 1.25 \\
\hline$(1,7)$ & 3 & 169.3 & 0.93 & 0.94 & 0.81 & 0.11 & 0.12 & 0.05 & & 1.17 & 2.41 \\
\hline$(7,1)$ & 3 & 168.4 & 0.96 & 0.95 & 0.81 & 0.10 & 0.12 & 0.05 & & 1.16 & 2.43 \\
\hline$(5,4)$ & 3.1 & 170.5 & 0.81 & 0.93 & 0.74 & 0.14 & 0.12 & 0.19 & 6.90 & 1.26 & 0.61 \\
\hline
\end{tabular}




\begin{tabular}{|c|c|c|c|c|c|c|c|c|c|c|c|}
\hline 1 & 2 & 3 & 4 & 5 & 6 & 7 & 8 & 9 & 10 & 11 & 12 \\
\hline$(4,5)$ & 3.1 & 170.5 & 0.89 & 0.96 & 0.73 & 0.13 & 0.11 & 0.19 & & 1.30 & 0.58 \\
\hline$(6,3)$ & 3.1 & 169.6 & 0.88 & 0.98 & 0.74 & 0.15 & 0.14 & 0.14 & 6.92 & 1.33 & 1.01 \\
\hline$(3,6)$ & 3.1 & 169.8 & 0.91 & 0.96 & 0.75 & 0.14 & 0.13 & 0.15 & & 1.28 & 0.89 \\
\hline$(2,7)$ & 3.2 & 170 & 0.91 & 0.97 & 0.80 & 0.15 & 0.15 & 0.11 & & 1.21 & 1.33 \\
\hline$(8,1)$ & 3.4 & 168.9 & 0.91 & 0.94 & 0.83 & 0.12 & 0.15 & 0.06 & 6.36 & 1.13 & 2.36 \\
\hline$(6,4)$ & 3.4 & 170.2 & 0.86 & 0.96 & 0.75 & 0.17 & 0.16 & 0.18 & 6.85 & 1.27 & 0.89 \\
\hline$(4,6)$ & 3.4 & 170.3 & 0.91 & 0.96 & 0.74 & 0.17 & 0.15 & 0.18 & & 1.30 & 0.82 \\
\hline$(7,3)$ & 3.5 & 170 & 0.93 & 0.93 & 0.78 & 0.15 & 0.14 & 0.14 & 6.75 & 1.20 & 1.07 \\
\hline$(3,7)$ & 3.5 & 170.4 & 0.91 & 0.92 & 0.76 & 0.15 & 0.15 & 0.14 & & 1.20 & 1.07 \\
\hline$(2,8)$ & 3.6 & 170.2 & 0.87 & 0.94 & 0.81 & 0.15 & 0.17 & 0.10 & & 1.16 & 1.63 \\
\hline$(5,6)$ & 3.7 & 170.8 & 0.81 & 0.93 & 0.72 & 0.16 & 0.12 & 0.20 & & 1.28 & 0.58 \\
\hline$(6,5)$ & 3.7 & 170.7 & 0.88 & 0.93 & 0.72 & 0.15 & 0.12 & 0.20 & 6.73 & 1.29 & 0.61 \\
\hline$(1,9)$ & 3.7 & 170.3 & 0.89 & 0.95 & 0.82 & 0.14 & 0.17 & 0.06 & & 1.16 & 2.66 \\
\hline$(7,4)$ & 3.8 & 170.6 & 0.89 & 0.96 & 0.76 & 0.17 & 0.16 & 0.17 & 6.74 & 1.27 & 0.95 \\
\hline$(4,7)$ & 3.8 & 170.7 & 0.94 & 1.00 & 0.76 & 0.17 & 0.16 & 0.17 & & 1.32 & 0.93 \\
\hline$(3,8)$ & 3.8 & 170.6 & 0.85 & 0.96 & 0.80 & 0.17 & 0.17 & 0.14 & & 1.21 & 1.27 \\
\hline$(8,3)$ & 3.8 & 170 & 0.91 & 0.95 & 0.79 & 0.17 & 0.17 & 0.14 & 6.62 & 1.20 & 1.25 \\
\hline$(2,9)$ & 4 & 170.6 & 0.87 & 0.92 & 0.81 & 0.16 & 0.18 & 0.12 & & 1.14 & 1.48 \\
\hline$(5,7)$ & 4.1 & 170.9 & 0.86 & 0.93 & 0.72 & 0.18 & 0.16 & 0.19 & & 1.28 & 0.84 \\
\hline$(10,1)$ & 4.1 & 169.6 & 0.81 & 0.90 & 0.82 & 0.14 & 0.18 & 0.07 & 6.09 & 1.10 & 2.47 \\
\hline$(8,4)$ & 4.1 & 170.3 & 0.91 & 0.94 & 0.77 & 0.18 & 0.17 & 0.17 & 6.64 & 1.23 & 1.03 \\
\hline$(4,8)$ & 4.1 & 170.5 & 0.88 & 0.98 & 0.77 & 0.18 & 0.17 & 0.17 & & 1.27 & 1.00 \\
\hline$(3,9)$ & 4.2 & 170.7 & 0.89 & 0.93 & 0.79 & 0.17 & 0.18 & 0.13 & & 1.19 & 1.43 \\
\hline$(9,3)$ & 4.2 & 169.8 & 0.90 & 0.94 & 0.78 & 0.17 & 0.18 & 0.13 & 6.40 & 1.19 & 1.35 \\
\hline$(2,10)$ & 4.3 & 170.4 & 0.93 & 0.92 & 0.80 & 0.16 & 0.20 & 0.10 & & 1.14 & 1.88 \\
\hline$(10,2)$ & 4.3 & 170 & 0.88 & 0.89 & 0.81 & 0.17 & 0.20 & 0.11 & 6.14 & 1.10 & 1.86 \\
\hline$(7,6)$ & 4.4 & 171.1 & 0.79 & 0.99 & 0.75 & 0.17 & 0.13 & 0.21 & 6.52 & 1.31 & 0.62 \\
\hline$(6,7)$ & 4.4 & 171.2 & 0.80 & 0.95 & 0.73 & 0.17 & 0.12 & 0.21 & & 1.30 & 0.58 \\
\hline$(5,8)$ & 4.4 & 171.1 & 0.84 & 0.92 & 0.74 & 0.19 & 0.17 & 0.19 & & 1.24 & 0.91 \\
\hline$(8,5)$ & 4.4 & 170.8 & 0.85 & 0.91 & 0.74 & 0.19 & 0.18 & 0.19 & 6.50 & 1.24 & 0.96 \\
\hline$(11,1)$ & 4.5 & 169.8 & 0.81 & 0.89 & 0.83 & 0.16 & 0.19 & 0.07 & 6.04 & 1.07 & 2.56 \\
\hline$(9,4)$ & 4.5 & 170.6 & 0.86 & 0.94 & 0.77 & 0.17 & 0.18 & 0.15 & 6.48 & 1.21 & 1.15 \\
\hline$(1,11)$ & 4.5 & 170.8 & 0.84 & 0.91 & 0.83 & 0.15 & 0.19 & 0.07 & & 1.10 & 2.67 \\
\hline$(4,9)$ & 4.5 & 170.9 & 0.85 & 0.93 & 0.77 & 0.17 & 0.18 & 0.15 & & 1.21 & 1.16 \\
\hline$(10,3)$ & 4.6 & 170.2 & 0.88 & 0.91 & 0.79 & 0.18 & 0.20 & 0.13 & 6.29 & 1.16 & 1.57 \\
\hline$(3,10)$ & 4.6 & 170.9 & 0.90 & 0.92 & 0.80 & 0.18 & 0.21 & 0.12 & & 1.16 & 1.71 \\
\hline$(2,11)$ & 4.7 & 170.8 & 0.87 & 0.94 & 0.82 & 0.17 & 0.20 & 0.11 & & 1.16 & 1.90 \\
\hline$(11,2)$ & 4.7 & 170 & 0.90 & 0.91 & 0.81 & 0.17 & 0.21 & 0.11 & 6.07 & 1.13 & 1.95 \\
\hline$(6,8)$ & 4.7 & 171 & 0.80 & 0.93 & 0.74 & 0.20 & 0.17 & 0.21 & & 1.26 & 0.81 \\
\hline$(8,6)$ & 4.7 & 170.9 & 0.86 & 0.92 & 0.72 & 0.20 & 0.18 & 0.22 & 6.40 & 1.27 & 0.83 \\
\hline$(5,9)$ & 4.8 & 171.1 & 0.83 & 0.91 & 0.75 & 0.18 & 0.17 & 0.18 & & 1.21 & 0.92 \\
\hline$(9,5)$ & 4.8 & 170.7 & 0.88 & 0.90 & 0.74 & 0.18 & 0.17 & 0.18 & 6.41 & 1.21 & 0.93 \\
\hline$(4,10)$ & 4.8 & 170.8 & 0.85 & 0.92 & 0.77 & 0.18 & 0.18 & 0.15 & & 1.19 & 1.17 \\
\hline$(10,4)$ & 4.8 & 170.4 & 0.89 & 0.92 & 0.77 & 0.19 & 0.19 & 0.15 & 6.26 & 1.20 & 1.28 \\
\hline$(1,12)$ & 4.9 & 170.8 & 0.86 & 0.87 & 0.82 & 0.16 & 0.20 & 0.07 & & 1.06 & 2.69 \\
\hline$(12,1)$ & 4.9 & 169.9 & 0.88 & 0.90 & 0.83 & 0.16 & 0.20 & 0.08 & 5.96 & 1.09 & 2.66 \\
\hline
\end{tabular}




\begin{tabular}{|c|c|c|c|c|c|c|c|c|c|c|c|}
\hline 1 & 2 & 3 & 4 & 5 & 6 & 7 & 8 & 9 & 10 & 11 & 12 \\
\hline$(11,3)$ & 5 & 170.3 & 0.88 & 0.93 & 0.80 & 0.19 & 0.20 & 0.14 & 6.09 & 1.16 & 1.43 \\
\hline$(3,11)$ & 5 & 170.9 & 0.87 & 0.93 & 0.81 & 0.19 & 0.19 & 0.14 & & 1.15 & 1.38 \\
\hline$(8,7)$ & 5 & 171.2 & 0.85 & 0.95 & 0.75 & 0.17 & 0.13 & 0.21 & 6.34 & 1.27 & 0.61 \\
\hline$(7,8)$ & 5 & 171.2 & 0.85 & 0.95 & 0.73 & 0.17 & 0.12 & 0.21 & & 1.30 & 0.59 \\
\hline$(9,6)$ & 5.1 & 170.8 & 0.81 & 0.94 & 0.73 & 0.20 & 0.18 & 0.20 & 6.33 & 1.29 & 0.89 \\
\hline$(6,9)$ & 5.1 & 171 & 0.82 & 0.94 & 0.73 & 0.20 & 0.19 & 0.20 & & 1.29 & 0.92 \\
\hline$(2,12)$ & 5.1 & 170.8 & 0.88 & 0.89 & 0.81 & 0.18 & 0.21 & 0.11 & & 1.11 & 1.90 \\
\hline$(12,2)$ & 5.1 & 170 & 0.89 & 0.93 & 0.82 & 0.17 & 0.20 & 0.11 & 6.01 & 1.13 & 1.85 \\
\hline$(5,10)$ & 5.1 & 171.2 & 0.86 & 0.97 & 0.76 & 0.18 & 0.17 & 0.18 & & 1.28 & 0.93 \\
\hline$(10,5)$ & 5.1 & 170.2 & 0.82 & 0.93 & 0.78 & 0.19 & 0.19 & 0.18 & 6.32 & 1.20 & 1.04 \\
\hline$(11,4)$ & 5.2 & 170.6 & 0.84 & 0.91 & 0.79 & 0.19 & 0.20 & 0.15 & 6.20 & 1.16 & 1.34 \\
\hline$(4,11)$ & 5.2 & 171.1 & 0.86 & 0.90 & 0.78 & 0.19 & 0.20 & 0.15 & & 1.16 & 1.36 \\
\hline$(1,13)$ & 5.2 & 170.9 & 0.88 & 0.90 & 0.83 & 0.16 & 0.21 & 0.08 & & 1.09 & 2.56 \\
\hline$(13,1)$ & 5.2 & 170 & 0.88 & 0.91 & 0.83 & 0.17 & 0.20 & 0.08 & 5.87 & 1.09 & 2.56 \\
\hline$(3,12)$ & 5.3 & 170.7 & 0.82 & 0.92 & 0.81 & 0.19 & 0.20 & 0.13 & & 1.13 & 1.61 \\
\hline$(7,9)$ & 5.4 & 171.3 & 0.84 & 0.94 & 0.74 & 0.20 & 0.19 & 0.21 & & 1.28 & 0.87 \\
\hline$(9,7)$ & 5.4 & 171 & 0.77 & 0.95 & 0.74 & 0.21 & 0.19 & 0.21 & 6.34 & 1.28 & 0.89 \\
\hline$(6,10)$ & 5.4 & 171.1 & 0.82 & 0.94 & 0.74 & 0.20 & 0.18 & 0.20 & & 1.26 & 0.94 \\
\hline$(10,6)$ & 5.4 & 170.9 & 0.83 & 0.96 & 0.75 & 0.20 & 0.19 & 0.19 & 6.37 & 1.29 & 0.99 \\
\hline$(13,2)$ & 5.5 & 170.2 & 0.85 & 0.90 & 0.82 & 0.19 & 0.21 & 0.11 & 5.91 & 1.10 & 1.93 \\
\hline$(2,13)$ & 5.5 & 171.1 & 0.90 & 0.90 & 0.81 & 0.19 & 0.21 & 0.11 & & 1.10 & 1.89 \\
\hline$(11,5)$ & 5.5 & 170.8 & 0.85 & 0.94 & 0.78 & 0.18 & 0.18 & 0.16 & 6.18 & 1.21 & 1.12 \\
\hline$(5,11)$ & 5.5 & 171.2 & 0.85 & 0.95 & 0.78 & 0.18 & 0.18 & 0.16 & & 1.22 & 1.11 \\
\hline$(12,4)$ & 5.6 & 170.3 & 0.87 & 0.87 & 0.77 & 0.19 & 0.20 & 0.15 & 6.07 & 1.12 & 1.39 \\
\hline$(4,12)$ & 5.6 & 170.7 & 0.81 & 0.88 & 0.80 & 0.19 & 0.20 & 0.14 & & 1.11 & 1.40 \\
\hline$(1,14)$ & 5.6 & 171.1 & 0.90 & 0.89 & 0.82 & 0.17 & 0.20 & 0.08 & & 1.08 & 2.50 \\
\hline$(8,9)$ & 5.7 & 171.2 & 0.82 & 0.93 & 0.73 & 0.17 & 0.14 & 0.21 & & 1.27 & 0.65 \\
\hline$(9,8)$ & 5.7 & 171.2 & 0.84 & 0.89 & 0.72 & 0.17 & 0.12 & 0.21 & 6.36 & 1.23 & 0.59 \\
\hline$(3,13)$ & 5.7 & 171.1 & 0.85 & 0.91 & 0.81 & 0.19 & 0.20 & 0.14 & & 1.13 & 1.46 \\
\hline$(13,3)$ & 5.7 & 170.4 & 0.87 & 0.89 & 0.80 & 0.19 & 0.21 & 0.14 & 6.10 & 1.11 & 1.47 \\
\hline$(10,7)$ & 5.7 & 171.1 & 0.80 & 0.94 & 0.75 & 0.20 & 0.20 & 0.21 & 6.40 & 1.25 & 0.95 \\
\hline$(7,10)$ & 5.7 & 171.2 & 0.82 & 0.94 & 0.74 & 0.19 & 0.18 & 0.21 & & 1.27 & 0.86 \\
\hline$(6,11)$ & 5.8 & 171.3 & 0.85 & 0.94 & 0.76 & 0.19 & 0.18 & 0.18 & & 1.23 & 0.97 \\
\hline$(11,6)$ & 5.8 & 170.9 & 0.84 & 0.95 & 0.76 & 0.19 & 0.18 & 0.18 & 6.37 & 1.25 & 0.97 \\
\hline$(2,14)$ & 5.9 & 170.8 & 0.85 & 0.88 & 0.82 & 0.18 & 0.22 & 0.11 & & 1.08 & 2.08 \\
\hline$(14,2)$ & 5.9 & 169.9 & 0.90 & 0.89 & 0.82 & 0.19 & 0.22 & 0.11 & 5.78 & 1.09 & 1.96 \\
\hline$(12,5)$ & 5.9 & 170.8 & 0.84 & 0.92 & 0.78 & 0.20 & 0.20 & 0.17 & 6.25 & 1.18 & 1.18 \\
\hline$(13,4)$ & 6 & 170.6 & 0.86 & 0.88 & 0.79 & 0.19 & 0.22 & 0.14 & 6.08 & 1.11 & 1.62 \\
\hline$(4,13)$ & 6 & 171.2 & 0.79 & 0.93 & 0.81 & 0.19 & 0.20 & 0.14 & & 1.15 & 1.40 \\
\hline$(15,1)$ & 6 & 170.2 & 0.84 & 0.88 & 0.83 & 0.18 & 0.22 & 0.08 & 5.90 & 1.06 & 2.76 \\
\hline$(1,15)$ & 6 & 171.1 & 0.87 & 0.85 & 0.82 & 0.17 & 0.21 & 0.09 & & 1.04 & 2.44 \\
\hline$(10,8)$ & 6 & 171.1 & 0.85 & 0.94 & 0.74 & 0.21 & 0.18 & 0.22 & 6.41 & 1.27 & 0.82 \\
\hline$(11,7)$ & 6.1 & 171.1 & 0.81 & 0.96 & 0.75 & 0.20 & 0.19 & 0.20 & 6.38 & 1.29 & 0.94 \\
\hline$(7,11)$ & 6.1 & 171.2 & 0.82 & 0.93 & 0.74 & 0.20 & 0.19 & 0.20 & & 1.26 & 0.92 \\
\hline$(3,14)$ & 6.1 & 171.2 & 0.83 & 0.89 & 0.82 & 0.18 & 0.21 & 0.12 & & 1.09 & 1.73 \\
\hline
\end{tabular}




\begin{tabular}{|c|c|c|c|c|c|c|c|c|c|c|c|}
\hline 1 & 2 & 3 & 4 & 5 & 6 & 7 & 8 & 9 & 10 & 11 & 12 \\
\hline$(14,3)$ & 6.1 & 170.5 & 0.85 & 0.89 & 0.81 & 0.18 & 0.20 & 0.12 & 6.10 & 1.10 & 1.65 \\
\hline$(6,12)$ & 6.1 & 171.1 & 0.81 & 0.94 & 0.78 & 0.20 & 0.18 & 0.18 & & 1.21 & 1.00 \\
\hline$(12,6)$ & 6.1 & 170.9 & 0.83 & 0.93 & 0.78 & 0.20 & 0.19 & 0.18 & 6.42 & 1.20 & 1.01 \\
\hline$(5,13)$ & 6.2 & 171.2 & 0.86 & 0.95 & 0.79 & 0.20 & 0.20 & 0.16 & & 1.21 & 1.23 \\
\hline$(13,5)$ & 6.2 & 170.7 & 0.85 & 0.92 & 0.78 & 0.20 & 0.19 & 0.17 & 6.28 & 1.18 & 1.17 \\
\hline$(2,15)$ & 6.2 & 171.1 & 0.84 & 0.86 & 0.81 & 0.19 & 0.22 & 0.11 & & 1.06 & 1.92 \\
\hline$(15,2)$ & 6.2 & 170.3 & 0.84 & 0.88 & 0.82 & 0.19 & 0.22 & 0.11 & 5.96 & 1.07 & 1.93 \\
\hline$(14,4)$ & 6.3 & 170.6 & 0.83 & 0.89 & 0.80 & 0.19 & 0.20 & 0.14 & 6.19 & 1.12 & 1.43 \\
\hline$(4,14)$ & 6.3 & 171 & 0.85 & 0.89 & 0.80 & 0.20 & 0.21 & 0.14 & & 1.12 & 1.57 \\
\hline$(9,10)$ & 6.4 & 171.3 & 0.83 & 0.93 & 0.74 & 0.17 & 0.13 & 0.21 & & 1.26 & 0.60 \\
\hline$(10,9)$ & 6.4 & 171.2 & 0.83 & 0.94 & 0.73 & 0.17 & 0.13 & 0.22 & 6.42 & 1.29 & 0.58 \\
\hline$(16,1)$ & 6.4 & 170.2 & 0.86 & 0.87 & 0.82 & 0.17 & 0.23 & 0.08 & 6.03 & 1.06 & 2.74 \\
\hline$(8,11)$ & 6.4 & 171.3 & 0.84 & 0.91 & 0.74 & 0.21 & 0.18 & 0.21 & & 1.24 & 0.84 \\
\hline$(12,7)$ & 6.4 & 171 & 0.83 & 0.96 & 0.75 & 0.20 & 0.19 & 0.20 & 6.38 & 1.27 & 0.95 \\
\hline$(7,12)$ & 6.4 & 171.3 & 0.80 & 0.93 & 0.75 & 0.20 & 0.19 & 0.20 & & 1.25 & 0.95 \\
\hline$(15,3)$ & 6.5 & 170.3 & 0.86 & 0.87 & 0.80 & 0.19 & 0.23 & 0.12 & 6.08 & 1.08 & 1.95 \\
\hline$(3,15)$ & 6.5 & 171.2 & 0.84 & 0.89 & 0.81 & 0.19 & 0.21 & 0.12 & & 1.09 & 1.77 \\
\hline$(13,6)$ & 6.5 & 170.8 & 0.82 & 0.94 & 0.78 & 0.19 & 0.18 & 0.17 & 6.40 & 1.21 & 1.09 \\
\hline$(6,13)$ & 6.5 & 171.2 & 0.83 & 0.92 & 0.77 & 0.19 & 0.19 & 0.17 & & 1.21 & 1.11 \\
\hline$(14,5)$ & 6.6 & 170.7 & 0.84 & 0.92 & 0.79 & 0.20 & 0.21 & 0.15 & 6.34 & 1.17 & 1.44 \\
\hline$(5,14)$ & 6.6 & 171.3 & 0.84 & 0.93 & 0.79 & 0.20 & 0.22 & 0.15 & & 1.18 & 1.41 \\
\hline$(2,16)$ & 6.6 & 171.1 & 0.84 & 0.85 & 0.81 & 0.20 & 0.23 & 0.11 & & 1.05 & 2.12 \\
\hline$(16,2)$ & 6.6 & 170.3 & 0.87 & 0.88 & 0.81 & 0.19 & 0.24 & 0.11 & 6.05 & 1.09 & 2.16 \\
\hline$(11,9)$ & 6.7 & 171.2 & 0.84 & 0.94 & 0.73 & 0.21 & 0.19 & 0.22 & 6.45 & 1.28 & 0.84 \\
\hline$(4,15)$ & 6.7 & 171.3 & 0.84 & 0.89 & 0.79 & 0.20 & 0.21 & 0.15 & & 1.13 & 1.41 \\
\hline$(15,4)$ & 6.7 & 170.6 & 0.86 & 0.88 & 0.80 & 0.21 & 0.21 & 0.15 & 6.17 & 1.09 & 1.44 \\
\hline$(12,8)$ & 6.7 & 171.1 & 0.82 & 0.93 & 0.74 & 0.21 & 0.19 & 0.21 & 6.38 & 1.25 & 0.89 \\
\hline$(8,12)$ & 6.7 & 171.3 & 0.85 & 0.93 & 0.75 & 0.20 & 0.18 & 0.21 & & 1.24 & 0.88 \\
\hline$(1,17)$ & 6.8 & 171.3 & 0.83 & 0.86 & 0.83 & 0.17 & 0.23 & 0.09 & & 1.04 & 2.61 \\
\hline$(13,7)$ & 6.8 & 171 & 0.84 & 0.93 & 0.77 & 0.19 & 0.18 & 0.18 & 6.39 & 1.22 & 0.99 \\
\hline$(7,13)$ & 6.8 & 171.3 & 0.83 & 0.94 & 0.75 & 0.19 & 0.18 & 0.18 & & 1.26 & 0.98 \\
\hline$(3,16)$ & 6.8 & 171.2 & 0.83 & 0.89 & 0.82 & 0.19 & 0.23 & 0.12 & & 1.09 & 1.89 \\
\hline$(16,3)$ & 6.8 & 170.5 & 0.84 & 0.86 & 0.81 & 0.19 & 0.22 & 0.12 & 6.14 & 1.06 & 1.87 \\
\hline$(6,14)$ & 6.9 & 171.3 & 0.83 & 0.94 & 0.77 & 0.20 & 0.19 & 0.17 & & 1.21 & 1.14 \\
\hline$(14,6)$ & 6.9 & 170.8 & 0.85 & 0.90 & 0.78 & 0.20 & 0.19 & 0.17 & 6.32 & 1.15 & 1.11 \\
\hline$(15,5)$ & 7 & 170.3 & 0.86 & 0.93 & 0.79 & 0.20 & 0.22 & 0.15 & 6.21 & 1.17 & 1.45 \\
\hline$(5,15)$ & 7 & 171.2 & 0.83 & 0.92 & 0.80 & 0.20 & 0.20 & 0.15 & & 1.16 & 1.30 \\
\hline$(2,17)$ & 7 & 171.2 & 0.83 & 0.87 & 0.83 & 0.20 & 0.24 & 0.11 & & 1.04 & 2.20 \\
\hline$(17,2)$ & 7 & 170.4 & 0.84 & 0.86 & 0.82 & 0.19 & 0.23 & 0.11 & 6.03 & 1.04 & 2.04 \\
\hline$(10,11)$ & 7 & 171.3 & 0.83 & 0.95 & 0.74 & 0.18 & 0.13 & 0.22 & & 1.28 & 0.60 \\
\hline$(11,10)$ & 7 & 171.2 & 0.82 & 0.92 & 0.73 & 0.17 & 0.12 & 0.22 & 6.45 & 1.26 & 0.57 \\
\hline$(9,12)$ & 7 & 171.1 & 0.80 & 0.91 & 0.73 & 0.21 & 0.19 & 0.22 & & 1.25 & 0.84 \\
\hline$(12,9)$ & 7 & 170.9 & 0.83 & 0.92 & 0.73 & 0.21 & 0.18 & 0.22 & 6.44 & 1.26 & 0.83 \\
\hline$(4,16)$ & 7.1 & 171.1 & 0.83 & 0.92 & 0.82 & 0.19 & 0.21 & 0.13 & & 1.12 & 1.59 \\
\hline$(8,13)$ & 7.1 & 171.3 & 0.84 & 0.91 & 0.74 & 0.21 & 0.19 & 0.20 & & 1.23 & 0.94 \\
\hline
\end{tabular}




\begin{tabular}{|c|c|c|c|c|c|c|c|c|c|c|c|}
\hline 1 & 2 & 3 & 4 & 5 & 6 & 7 & 8 & 9 & 10 & 11 & 12 \\
\hline$(13,8)$ & 7.1 & 171 & 0.84 & 0.93 & 0.74 & 0.21 & 0.19 & 0.20 & 6.41 & 1.27 & 0.95 \\
\hline$(18,1)$ & 7.1 & 170.3 & 0.85 & 0.85 & 0.82 & 0.18 & 0.23 & 0.08 & 6.04 & 1.03 & 2.75 \\
\hline$(7,14)$ & 7.2 & 171.1 & 0.87 & 0.93 & 0.78 & 0.20 & 0.19 & 0.18 & & 1.20 & 1.02 \\
\hline$(14,7)$ & 7.2 & 170.8 & 0.83 & 0.92 & 0.77 & 0.21 & 0.20 & 0.19 & 6.47 & 1.18 & 1.07 \\
\hline$(3,17)$ & 7.2 & 171.3 & 0.83 & 0.91 & 0.82 & 0.20 & 0.23 & 0.12 & & 1.11 & 2.01 \\
\hline$(17,3)$ & 7.2 & 170.5 & 0.83 & 0.87 & 0.82 & 0.20 & 0.23 & 0.12 & 6.14 & 1.06 & 1.93 \\
\hline$(15,6)$ & 7.2 & 170.6 & 0.83 & 0.92 & 0.78 & 0.20 & 0.20 & 0.17 & 6.37 & 1.18 & 1.20 \\
\hline$(6,15)$ & 7.2 & 171.3 & 0.83 & 0.91 & 0.78 & 0.19 & 0.20 & 0.17 & & 1.17 & 1.15 \\
\hline$(5,16)$ & 7.3 & 171.3 & 0.83 & 0.90 & 0.80 & 0.20 & 0.22 & 0.14 & & 1.13 & 1.55 \\
\hline$(16,5)$ & 7.3 & 170.7 & 0.83 & 0.91 & 0.80 & 0.20 & 0.22 & 0.14 & 6.28 & 1.13 & 1.54 \\
\hline$(12,10)$ & 7.4 & 171 & 0.85 & 0.94 & 0.74 & 0.21 & 0.18 & 0.23 & 6.53 & 1.26 & 0.77 \\
\hline$(10,12)$ & 7.4 & 171.3 & 0.84 & 0.92 & 0.73 & 0.21 & 0.18 & 0.23 & & 1.27 & 0.80 \\
\hline$(2,18)$ & 7.4 & 171.3 & 0.85 & 0.86 & 0.82 & 0.19 & 0.23 & 0.11 & & 1.05 & 2.09 \\
\hline$(18,2)$ & 7.4 & 170.2 & 0.84 & 0.87 & 0.82 & 0.20 & 0.24 & 0.11 & 6.05 & 1.06 & 2.13 \\
\hline$(9,13)$ & 7.4 & 171.3 & 0.84 & 0.92 & 0.73 & 0.21 & 0.18 & 0.21 & & 1.25 & 0.87 \\
\hline$(14,8)$ & 7.4 & 171 & 0.86 & 0.93 & 0.76 & 0.21 & 0.19 & 0.20 & 6.42 & 1.22 & 0.96 \\
\hline$(8,14)$ & 7.4 & 171.2 & 0.86 & 0.95 & 0.75 & 0.21 & 0.19 & 0.20 & & 1.25 & 0.96 \\
\hline$(4,17)$ & 7.5 & 171.3 & 0.87 & 0.90 & 0.81 & 0.21 & 0.22 & 0.14 & & 1.11 & 1.56 \\
\hline$(17,4)$ & 7.5 & 170.6 & 0.85 & 0.90 & 0.81 & 0.21 & 0.22 & 0.15 & 6.18 & 1.10 & 1.52 \\
\hline$(7,15)$ & 7.5 & 171.4 & 0.84 & 0.93 & 0.77 & 0.20 & 0.18 & 0.17 & & 1.21 & 1.08 \\
\hline$(15,7)$ & 7.5 & 170.9 & 0.86 & 0.92 & 0.78 & 0.19 & 0.19 & 0.17 & 6.35 & 1.18 & 1.09 \\
\hline$(1,19)$ & 7.5 & 171.3 & 0.86 & 0.86 & 0.84 & 0.18 & 0.21 & 0.08 & & 1.03 & 2.63 \\
\hline$(19,1)$ & 7.5 & 170.3 & 0.84 & 0.87 & 0.82 & 0.19 & 0.23 & 0.09 & 6.07 & 1.05 & 2.71 \\
\hline$(3,18)$ & 7.6 & 171.2 & 0.86 & 0.89 & 0.82 & 0.20 & 0.21 & 0.12 & & 1.08 & 1.80 \\
\hline$(18,3)$ & 7.6 & 170.3 & 0.85 & 0.88 & 0.82 & 0.19 & 0.22 & 0.12 & 6.16 & 1.08 & 1.81 \\
\hline$(16,6)$ & 7.6 & 170.8 & 0.82 & 0.91 & 0.80 & 0.20 & 0.21 & 0.16 & 6.32 & 1.14 & 1.30 \\
\hline$(6,16)$ & 7.6 & 171.3 & 0.83 & 0.91 & 0.79 & 0.20 & 0.20 & 0.16 & & 1.15 & 1.25 \\
\hline$(12,11)$ & 7.7 & 171.2 & 0.89 & 0.94 & 0.74 & 0.17 & 0.12 & 0.21 & 6.49 & 1.27 & 0.55 \\
\hline$(11,12)$ & 7.7 & 171.3 & 0.82 & 0.96 & 0.74 & 0.18 & 0.14 & 0.22 & & 1.30 & 0.64 \\
\hline$(13,10)$ & 7.7 & 171.2 & 0.87 & 0.91 & 0.74 & 0.21 & 0.18 & 0.22 & 6.42 & 1.23 & 0.83 \\
\hline$(10,13)$ & 7.7 & 171.4 & 0.85 & 0.93 & 0.74 & 0.21 & 0.18 & 0.22 & & 1.26 & 0.79 \\
\hline$(17,5)$ & 7.7 & 170.7 & 0.86 & 0.92 & 0.82 & 0.20 & 0.22 & 0.14 & 6.24 & 1.12 & 1.53 \\
\hline$(5,17)$ & 7.7 & 171.3 & 0.82 & 0.88 & 0.80 & 0.20 & 0.23 & 0.14 & & 1.11 & 1.58 \\
\hline$(14,9)$ & 7.7 & 171.1 & 0.82 & 0.92 & 0.74 & 0.20 & 0.19 & 0.21 & 6.42 & 1.24 & 0.92 \\
\hline$(9,14)$ & 7.7 & 171.3 & 0.85 & 0.91 & 0.74 & 0.20 & 0.19 & 0.21 & & 1.23 & 0.91 \\
\hline$(19,2)$ & 7.8 & 170.5 & 0.86 & 0.87 & 0.83 & 0.20 & 0.25 & 0.11 & 6.10 & 1.04 & 2.28 \\
\hline$(2,19)$ & 7.8 & 171.3 & 0.86 & 0.86 & 0.81 & 0.20 & 0.24 & 0.11 & & 1.06 & 2.16 \\
\hline$(8,15)$ & 7.8 & 171.3 & 0.86 & 0.92 & 0.76 & 0.20 & 0.19 & 0.18 & & 1.22 & 1.02 \\
\hline$(15,8)$ & 7.8 & 171 & 0.84 & 0.93 & 0.76 & 0.19 & 0.18 & 0.19 & 6.39 & 1.23 & 0.93 \\
\hline$(18,4)$ & 7.8 & 170.5 & 0.84 & 0.87 & 0.81 & 0.20 & 0.22 & 0.13 & 6.17 & 1.08 & 1.69 \\
\hline$(4,18)$ & 7.8 & 171.2 & 0.84 & 0.89 & 0.80 & 0.20 & 0.23 & 0.13 & & 1.10 & 1.80 \\
\hline$(16,7)$ & 7.9 & 170.9 & 0.84 & 0.92 & 0.78 & 0.20 & 0.21 & 0.18 & 6.28 & 1.18 & 1.20 \\
\hline$(7,16)$ & 7.9 & 171.4 & 0.84 & 0.89 & 0.77 & 0.20 & 0.20 & 0.18 & & 1.15 & 1.12 \\
\hline$(20,1)$ & 7.9 & 170.3 & 0.84 & 0.87 & 0.83 & 0.18 & 0.23 & 0.08 & 6.07 & 1.04 & 2.80 \\
\hline$(1,20)$ & 7.9 & 171.3 & 0.83 & 0.87 & 0.83 & 0.18 & 0.23 & 0.09 & & 1.05 & 2.66 \\
\hline
\end{tabular}




\begin{tabular}{|c|c|c|c|c|c|c|c|c|c|c|c|}
\hline 1 & 2 & 3 & 4 & 5 & 6 & 7 & 8 & 9 & 10 & 11 & 12 \\
\hline$(6,17)$ & 8 & 171.3 & 0.83 & 0.88 & 0.79 & 0.21 & 0.22 & 0.15 & & 1.11 & 1.46 \\
\hline$(17,6)$ & 8 & 170.8 & 0.84 & 0.89 & 0.79 & 0.21 & 0.21 & 0.16 & 6.30 & 1.13 & 1.36 \\
\hline$(3,19)$ & 8 & 171.3 & 0.85 & 0.88 & 0.82 & 0.20 & 0.22 & 0.11 & & 1.07 & 1.97 \\
\hline$(19,3)$ & 8 & 170.6 & 0.86 & 0.86 & 0.81 & 0.20 & 0.23 & 0.12 & 6.12 & 1.06 & 1.90 \\
\hline$(11,13)$ & 8 & 171.3 & 0.83 & 0.92 & 0.73 & 0.21 & 0.17 & 0.23 & & 1.26 & 0.74 \\
\hline$(13,11)$ & 8 & 171.2 & 0.84 & 0.95 & 0.74 & 0.21 & 0.18 & 0.23 & 6.35 & 1.28 & 0.78 \\
\hline$(14,10)$ & 8.1 & 171.2 & 0.87 & 0.93 & 0.74 & 0.21 & 0.18 & 0.22 & 6.44 & 1.25 & 0.80 \\
\hline$(18,5)$ & 8.1 & 170.8 & 0.84 & 0.88 & 0.80 & 0.20 & 0.21 & 0.14 & 6.17 & 1.09 & 1.52 \\
\hline$(5,18)$ & 8.1 & 171.4 & 0.82 & 0.87 & 0.80 & 0.20 & 0.21 & 0.14 & & 1.09 & 1.48 \\
\hline$(9,15)$ & 8.1 & 171.3 & 0.88 & 0.91 & 0.75 & 0.20 & 0.19 & 0.20 & & 1.21 & 0.92 \\
\hline$(15,9)$ & 8.1 & 171.1 & 0.85 & 0.91 & 0.75 & 0.21 & 0.19 & 0.21 & 6.52 & 1.21 & 0.90 \\
\hline$(2,20)$ & 8.1 & 171.3 & 0.84 & 0.87 & 0.83 & 0.20 & 0.25 & 0.11 & & 1.05 & 2.21 \\
\hline$(20,2)$ & 8.1 & 170.3 & 0.82 & 0.89 & 0.83 & 0.20 & 0.23 & 0.11 & 6.06 & 1.07 & 2.10 \\
\hline$(16,8)$ & 8.2 & 170.8 & 0.84 & 0.91 & 0.77 & 0.20 & 0.19 & 0.19 & 6.46 & 1.18 & 1.02 \\
\hline$(8,16)$ & 8.2 & 171 & 0.83 & 0.89 & 0.76 & 0.21 & 0.19 & 0.19 & & 1.18 & 1.00 \\
\hline$(4,19)$ & 8.2 & 171.4 & 0.83 & 0.87 & 0.81 & 0.19 & 0.23 & 0.12 & & 1.07 & 1.83 \\
\hline$(19,4)$ & 8.2 & 170.6 & 0.86 & 0.88 & 0.81 & 0.20 & 0.22 & 0.13 & 6.13 & 1.09 & 1.69 \\
\hline$(7,17)$ & 8.3 & 171.4 & 0.82 & 0.89 & 0.78 & 0.21 & 0.21 & 0.17 & & 1.15 & 1.23 \\
\hline$(17,7)$ & 8.3 & 170.8 & 0.87 & 0.91 & 0.78 & 0.21 & 0.20 & 0.17 & 6.39 & 1.17 & 1.14 \\
\hline$(13,12)$ & 8.3 & 171.2 & 0.84 & 0.91 & 0.73 & 0.17 & 0.12 & 0.21 & 6.50 & 1.25 & 0.55 \\
\hline$(12,13)$ & 8.3 & 171.3 & 0.84 & 0.89 & 0.72 & 0.17 & 0.13 & 0.22 & & 1.24 & 0.61 \\
\hline$(6,18)$ & 8.4 & 171.2 & 0.82 & 0.90 & 0.80 & 0.19 & 0.21 & 0.16 & & 1.13 & 1.32 \\
\hline$(18,6)$ & 8.4 & 170.8 & 0.84 & 0.91 & 0.79 & 0.20 & 0.22 & 0.15 & 6.32 & 1.15 & 1.43 \\
\hline$(20,3)$ & 8.4 & 170.5 & 0.84 & 0.85 & 0.82 & 0.20 & 0.23 & 0.12 & 6.18 & 1.04 & 1.83 \\
\hline$(3,20)$ & 8.4 & 171.3 & 0.84 & 0.87 & 0.81 & 0.20 & 0.23 & 0.12 & & 1.07 & 1.91 \\
\hline$(11,14)$ & 8.4 & 171.3 & 0.84 & 0.90 & 0.74 & 0.21 & 0.18 & 0.22 & & 1.22 & 0.83 \\
\hline$(14,11)$ & 8.4 & 171.2 & 0.82 & 0.93 & 0.75 & 0.21 & 0.18 & 0.22 & 6.37 & 1.25 & 0.81 \\
\hline$(10,15)$ & 8.4 & 171.2 & 0.85 & 0.93 & 0.75 & 0.21 & 0.19 & 0.21 & & 1.24 & 0.88 \\
\hline$(15,10)$ & 8.4 & 171 & 0.84 & 0.92 & 0.74 & 0.21 & 0.18 & 0.21 & 6.52 & 1.24 & 0.83 \\
\hline$(9,16)$ & 8.5 & 171.4 & 0.82 & 0.92 & 0.76 & 0.20 & 0.19 & 0.20 & & 1.22 & 0.95 \\
\hline$(5,19)$ & 8.5 & 171.4 & 0.84 & 0.88 & 0.80 & 0.21 & 0.21 & 0.15 & & 1.10 & 1.43 \\
\hline$(19,5)$ & 8.5 & 170.7 & 0.83 & 0.87 & 0.80 & 0.21 & 0.20 & 0.15 & 6.24 & 1.09 & 1.33 \\
\hline$(17,8)$ & 8.5 & 170.9 & 0.84 & 0.92 & 0.77 & 0.21 & 0.17 & 0.17 & 6.45 & 1.20 & 1.02 \\
\hline$(8,17)$ & 8.5 & 171.4 & 0.85 & 0.89 & 0.76 & 0.20 & 0.19 & 0.17 & & 1.17 & 1.11 \\
\hline$(20,4)$ & 8.6 & 170.3 & 0.85 & 0.87 & 0.82 & 0.20 & 0.22 & 0.13 & 6.25 & 1.06 & 1.72 \\
\hline$(4,20)$ & 8.6 & 171.2 & 0.84 & 0.89 & 0.81 & 0.20 & 0.22 & 0.13 & & 1.10 & 1.73 \\
\hline$(18,7)$ & 8.6 & 170.9 & 0.86 & 0.90 & 0.78 & 0.20 & 0.19 & 0.17 & 6.34 & 1.16 & 1.12 \\
\hline$(7,18)$ & 8.6 & 171.4 & 0.86 & 0.90 & 0.78 & 0.21 & 0.20 & 0.17 & & 1.14 & 1.18 \\
\hline$(14,12)$ & 8.7 & 171.2 & 0.87 & 0.94 & 0.74 & 0.21 & 0.18 & 0.23 & 6.47 & 1.26 & 0.76 \\
\hline$(12,14)$ & 8.7 & 171.2 & 0.83 & 0.90 & 0.73 & 0.21 & 0.17 & 0.23 & & 1.24 & 0.73 \\
\hline$(15,11)$ & 8.7 & 171.2 & 0.88 & 0.93 & 0.74 & 0.21 & 0.19 & 0.22 & 6.52 & 1.26 & 0.86 \\
\hline$(11,15)$ & 8.7 & 171.3 & 0.86 & 0.92 & 0.74 & 0.21 & 0.19 & 0.22 & & 1.24 & 0.87 \\
\hline$(6,19)$ & 8.7 & 171.4 & 0.86 & 0.89 & 0.79 & 0.21 & 0.22 & 0.15 & & 1.12 & 1.52 \\
\hline$(19,6)$ & 8.7 & 170.8 & 0.85 & 0.88 & 0.79 & 0.21 & 0.23 & 0.15 & 6.25 & 1.12 & 1.54 \\
\hline$(16,10)$ & 8.8 & 171.1 & 0.85 & 0.93 & 0.76 & 0.21 & 0.18 & 0.21 & 6.46 & 1.22 & 0.87 \\
\hline
\end{tabular}




\begin{tabular}{|c|c|c|c|c|c|c|c|c|c|c|c|}
\hline 1 & 2 & 3 & 4 & 5 & 6 & 7 & 8 & 9 & 10 & 11 & 12 \\
\hline$(10,16)$ & 8.8 & 171.4 & 0.86 & 0.92 & 0.75 & 0.21 & 0.18 & 0.21 & & 1.22 & 0.89 \\
\hline$(17,9)$ & 8.8 & 171 & 0.86 & 0.93 & 0.76 & 0.20 & 0.19 & 0.18 & 6.45 & 1.22 & 1.06 \\
\hline$(9,17)$ & 8.8 & 171.4 & 0.84 & 0.92 & 0.75 & 0.19 & 0.19 & 0.19 & & 1.22 & 1.01 \\
\hline$(5,20)$ & 8.8 & 171.1 & 0.83 & 0.88 & 0.81 & 0.20 & 0.21 & 0.14 & & 1.09 & 1.51 \\
\hline$(20,5)$ & 8.8 & 170.6 & 0.84 & 0.87 & 0.80 & 0.20 & 0.23 & 0.14 & 6.25 & 1.09 & 1.69 \\
\hline$(18,8)$ & 8.9 & 170.9 & 0.86 & 0.91 & 0.78 & 0.20 & 0.20 & 0.18 & 6.39 & 1.17 & 1.16 \\
\hline$(8,18)$ & 8.9 & 171.4 & 0.85 & 0.89 & 0.77 & 0.20 & 0.21 & 0.18 & & 1.15 & 1.16 \\
\hline$(19,7)$ & 9 & 170.9 & 0.83 & 0.88 & 0.79 & 0.20 & 0.20 & 0.16 & 6.45 & 1.12 & 1.30 \\
\hline$(7,19)$ & 9 & 171.4 & 0.86 & 0.90 & 0.79 & 0.20 & 0.21 & 0.16 & & 1.14 & 1.30 \\
\hline$(13,14)$ & 9 & 171.3 & 0.84 & 0.93 & 0.74 & 0.17 & 0.13 & 0.22 & & 1.26 & 0.60 \\
\hline$(14,13)$ & 9 & 171.3 & 0.85 & 0.90 & 0.73 & 0.17 & 0.12 & 0.22 & 6.59 & 1.25 & 0.55 \\
\hline$(15,12)$ & 9 & 171.2 & 0.87 & 0.94 & 0.74 & 0.21 & 0.18 & 0.22 & 6.53 & 1.26 & 0.81 \\
\hline$(12,15)$ & 9 & 171.3 & 0.85 & 0.92 & 0.74 & 0.21 & 0.18 & 0.23 & & 1.24 & 0.79 \\
\hline$(11,16)$ & 9.1 & 171.4 & 0.85 & 0.92 & 0.74 & 0.21 & 0.18 & 0.21 & & 1.24 & 0.85 \\
\hline$(16,11)$ & 9.1 & 171.2 & 0.83 & 0.91 & 0.74 & 0.20 & 0.17 & 0.21 & 6.43 & 1.23 & 0.82 \\
\hline$(6,20)$ & 9.1 & 171.4 & 0.84 & 0.89 & 0.80 & 0.20 & 0.21 & 0.15 & & 1.11 & 1.41 \\
\hline$(10,17)$ & 9.1 & 171.4 & 0.86 & 0.93 & 0.76 & 0.21 & 0.20 & 0.20 & & 1.23 & 0.98 \\
\hline$(17,10)$ & 9.1 & 171.1 & 0.84 & 0.93 & 0.76 & 0.21 & 0.19 & 0.21 & 6.54 & 1.22 & 0.94 \\
\hline$(18,9)$ & 9.2 & 170.7 & 0.84 & 0.90 & 0.77 & 0.20 & 0.20 & 0.19 & 6.37 & 1.17 & 1.06 \\
\hline$(9,18)$ & 9.2 & 170.9 & 0.83 & 0.90 & 0.77 & 0.21 & 0.19 & 0.19 & & 1.18 & 1.00 \\
\hline$(8,19)$ & 9.3 & 171.4 & 0.85 & 0.91 & 0.77 & 0.20 & 0.20 & 0.17 & & 1.17 & 1.16 \\
\hline$(19,8)$ & 9.3 & 170.9 & 0.85 & 0.89 & 0.77 & 0.20 & 0.20 & 0.17 & 6.47 & 1.15 & 1.18 \\
\hline$(13,15)$ & 9.4 & 171.4 & 0.83 & 0.92 & 0.74 & 0.20 & 0.17 & 0.23 & & 1.25 & 0.75 \\
\hline$(15,13)$ & 9.4 & 171.2 & 0.86 & 0.94 & 0.74 & 0.21 & 0.18 & 0.23 & 6.38 & 1.27 & 0.78 \\
\hline$(7,20)$ & 9.4 & 171.4 & 0.86 & 0.89 & 0.79 & 0.20 & 0.22 & 0.16 & & 1.13 & 1.43 \\
\hline$(20,7)$ & 9.4 & 170.8 & 0.84 & 0.88 & 0.78 & 0.21 & 0.21 & 0.16 & 6.41 & 1.12 & 1.35 \\
\hline$(16,12)$ & 9.4 & 171.1 & 0.82 & 0.90 & 0.73 & 0.20 & 0.17 & 0.22 & 6.53 & 1.23 & 0.77 \\
\hline$(11,17)$ & 9.4 & 171.4 & 0.84 & 0.93 & 0.75 & 0.20 & 0.17 & 0.21 & & 1.23 & 0.83 \\
\hline$(17,11)$ & 9.4 & 171.1 & 0.85 & 0.93 & 0.75 & 0.21 & 0.18 & 0.21 & 6.37 & 1.24 & 0.88 \\
\hline$(10,18)$ & 9.5 & 171.3 & 0.85 & 0.91 & 0.76 & 0.21 & 0.20 & 0.20 & & 1.20 & 1.00 \\
\hline$(18,10)$ & 9.5 & 171.1 & 0.85 & 0.91 & 0.76 & 0.21 & 0.19 & 0.20 & & 1.19 & 0.95 \\
\hline$(9,19)$ & 9.5 & 171.4 & 0.89 & 0.91 & 0.78 & 0.19 & 0.19 & 0.18 & & 1.18 & 1.06 \\
\hline$(19,9)$ & 9.5 & 171 & 0.86 & 0.93 & 0.77 & 0.20 & 0.20 & 0.18 & 6.37 & 1.20 & 1.10 \\
\hline$(20,8)$ & 9.6 & 170.9 & 0.84 & 0.89 & 0.77 & 0.20 & 0.21 & 0.17 & 6.29 & 1.15 & 1.28 \\
\hline$(14,15)$ & 9.7 & 171.4 & 0.88 & 0.93 & 0.74 & 0.17 & 0.13 & 0.21 & & 1.26 & 0.60 \\
\hline$(15,14)$ & 9.7 & 171.3 & 0.87 & 0.94 & 0.74 & 0.18 & 0.12 & 0.22 & 6.51 & 1.28 & 0.55 \\
\hline$(13,16)$ & 9.7 & 171.4 & 0.86 & 0.95 & 0.75 & 0.21 & 0.18 & 0.23 & & 1.27 & 0.81 \\
\hline$(16,13)$ & 9.7 & 171.3 & 0.84 & 0.95 & 0.75 & 0.21 & 0.18 & 0.23 & 6.50 & 1.27 & 0.80 \\
\hline$(12,17)$ & 9.7 & 171.4 & 0.83 & 0.92 & 0.75 & 0.21 & 0.18 & 0.22 & & 1.24 & 0.84 \\
\hline$(17,12)$ & 9.7 & 171.2 & 0.84 & 0.93 & 0.74 & 0.21 & 0.18 & 0.22 & 6.55 & 1.25 & 0.82 \\
\hline$(11,18)$ & 9.8 & 171.4 & 0.85 & 0.91 & 0.76 & 0.21 & 0.19 & 0.21 & & 1.20 & 0.91 \\
\hline$(18,11)$ & 9.8 & 171.1 & 0.84 & 0.92 & 0.75 & 0.21 & 0.18 & 0.21 & 6.51 & 1.23 & 0.88 \\
\hline$(19,10)$ & 9.8 & 171 & 0.85 & 0.91 & 0.76 & 0.19 & 0.18 & 0.19 & 6.44 & 1.20 & 0.97 \\
\hline$(9,20)$ & 9.9 & 171.4 & 0.84 & 0.90 & 0.77 & 0.20 & 0.19 & 0.18 & & 1.17 & 1.09 \\
\hline$(20,9)$ & 9.9 & 171 & 0.85 & 0.88 & 0.76 & 0.21 & 0.19 & 0.18 & 6.18 & 1.15 & 1.08 \\
\hline
\end{tabular}




\begin{tabular}{|c|c|c|c|c|c|c|c|c|c|c|c|}
\hline 1 & 2 & 3 & 4 & 5 & 6 & 7 & 8 & 9 & 10 & 11 & 12 \\
\hline$(14,16)$ & 10 & 171.3 & 0.85 & 0.92 & 0.73 & 0.21 & 0.18 & 0.23 & & 1.26 & 0.81 \\
\hline$(16,14)$ & 10 & 171.3 & 0.85 & 0.93 & 0.74 & 0.21 & 0.18 & 0.23 & 6.14 & 1.26 & 0.76 \\
\hline$(17,13)$ & 10 & 171.2 & 0.83 & 0.93 & 0.74 & 0.21 & 0.17 & 0.22 & 5.12 & 1.26 & 0.78 \\
\hline$(13,17)$ & 10 & 171.3 & 0.86 & 0.93 & 0.74 & 0.21 & 0.19 & 0.23 & & 1.26 & 0.82 \\
\hline$(12,18)$ & 10 & 171.1 & 0.86 & 0.92 & 0.75 & 0.20 & 0.17 & 0.21 & & 1.23 & 0.82 \\
\hline$(18,12)$ & 10 & 171 & 0.85 & 0.93 & 0.75 & 0.20 & 0.18 & 0.21 & 6.44 & 1.24 & 0.86 \\
\hline$(19,11)$ & 10 & 171.1 & 0.84 & 0.90 & 0.76 & 0.21 & 0.18 & 0.20 & 6.47 & 1.18 & 0.92 \\
\hline$(11,19)$ & 10 & 171.4 & 0.87 & 0.91 & 0.75 & 0.21 & 0.17 & 0.20 & & 1.20 & 0.85 \\
\hline$(10,20)$ & 10 & 170.9 & 0.86 & 0.91 & 0.76 & 0.20 & 0.18 & 0.19 & & 1.19 & 0.96 \\
\hline$(20,10)$ & 10 & 170.7 & 0.85 & 0.91 & 0.76 & 0.21 & 0.20 & 0.19 & 6.37 & 1.19 & 1.01 \\
\hline$(15,16)$ & 10 & 171.3 & 0.86 & 0.94 & 0.74 & 0.17 & 0.13 & 0.22 & & 1.27 & 0.61 \\
\hline$(16,15)$ & 10 & 171.3 & 0.88 & 0.94 & 0.73 & 0.17 & 0.14 & 0.22 & 6.53 & 1.29 & 0.64 \\
\hline$(17,14)$ & 10 & 171.3 & 0.91 & 0.94 & 0.75 & 0.21 & 0.19 & 0.23 & 6.30 & 1.27 & 0.82 \\
\hline$(14,17)$ & 10 & 171.4 & 0.87 & 0.90 & 0.73 & 0.21 & 0.18 & 0.23 & & 1.24 & 0.77 \\
\hline$(13,18)$ & 10 & 171.4 & 0.87 & 0.92 & 0.75 & 0.21 & 0.18 & 0.22 & & 1.23 & 0.80 \\
\hline$(19,12)$ & 10 & 171.2 & 0.86 & 0.95 & 0.76 & 0.21 & 0.18 & 0.21 & 6.41 & 1.25 & 0.86 \\
\hline$(12,19)$ & 10 & 171.4 & 0.86 & 0.92 & 0.75 & 0.21 & 0.18 & 0.21 & & 1.23 & 0.88 \\
\hline$(20,11)$ & 10 & 171.1 & 0.87 & 0.92 & 0.76 & 0.20 & 0.19 & 0.20 & & 1.21 & 0.99 \\
\hline$(11,20)$ & 10 & 171.4 & 0.83 & 0.92 & 0.76 & 0.21 & 0.20 & 0.19 & & 1.21 & 1.03 \\
\hline$(18,14)$ & 11 & 171.2 & 0.85 & 0.92 & 0.74 & 0.21 & 0.18 & 0.22 & 6.42 & 1.24 & 0.80 \\
\hline$(14,18)$ & 11 & 171.4 & 0.87 & 0.93 & 0.74 & 0.21 & 0.18 & 0.23 & & 1.25 & 0.77 \\
\hline$(13,19)$ & 11 & 171.4 & 0.86 & 0.94 & 0.75 & 0.20 & 0.19 & 0.21 & & 1.25 & 0.88 \\
\hline$(19,13)$ & 11 & 171.2 & 0.87 & 0.94 & 0.76 & 0.21 & 0.19 & 0.21 & 6.39 & 1.24 & 0.89 \\
\hline$(20,12)$ & 11 & 171 & 0.87 & 0.93 & 0.76 & 0.20 & 0.19 & 0.21 & 6.38 & 1.23 & 0.90 \\
\hline$(12,20)$ & 11 & 171.2 & 0.84 & 0.94 & 0.76 & 0.21 & 0.18 & 0.21 & & 1.24 & 0.88 \\
\hline$(17,16)$ & 11 & 171.3 & 0.83 & 0.94 & 0.73 & 0.17 & 0.13 & 0.21 & 6.42 & 1.28 & 0.62 \\
\hline$(16,17)$ & 11 & 171.3 & 0.85 & 0.93 & 0.73 & 0.17 & 0.11 & 0.22 & & 1.27 & 0.50 \\
\hline$(15,18)$ & 11 & 171.2 & 0.86 & 0.92 & 0.74 & 0.21 & 0.18 & 0.22 & & 1.25 & 0.81 \\
\hline$(18,15)$ & 11 & 171.1 & 0.90 & 0.97 & 0.75 & 0.21 & 0.19 & 0.23 & 6.44 & 1.29 & 0.84 \\
\hline$(14,19)$ & 11 & 171.4 & 0.86 & 0.94 & 0.74 & 0.21 & 0.19 & 0.22 & & 1.26 & 0.85 \\
\hline$(19,14)$ & 11 & 171.2 & 0.87 & 0.93 & 0.74 & 0.21 & 0.17 & 0.22 & 6.39 & 1.26 & 0.79 \\
\hline$(20,13)$ & 11 & 171.1 & 0.84 & 0.93 & 0.75 & 0.21 & 0.18 & 0.21 & 6.41 & 1.24 & 0.84 \\
\hline$(13,20)$ & 11 & 171.4 & 0.90 & 0.93 & 0.75 & 0.20 & 0.18 & 0.21 & & 1.24 & 0.85 \\
\hline$(16,18)$ & 11 & 171.4 & 0.85 & 0.93 & 0.74 & 0.21 & 0.18 & 0.23 & & 1.26 & 0.77 \\
\hline$(18,16)$ & 11 & 171.2 & 0.85 & 0.93 & 0.73 & 0.21 & 0.18 & 0.23 & 6.39 & 1.27 & 0.78 \\
\hline$(15,19)$ & 11 & 171.4 & 0.86 & 0.93 & 0.74 & 0.21 & 0.18 & 0.22 & & 1.26 & 0.80 \\
\hline$(19,15)$ & 11 & 171.2 & 0.86 & 0.92 & 0.74 & 0.21 & 0.17 & 0.22 & 6.39 & 1.24 & 0.77 \\
\hline$(18,17)$ & 12 & 171.3 & 0.85 & 0.92 & 0.74 & 0.17 & 0.11 & 0.22 & 6.39 & 1.25 & 0.52 \\
\hline$(17,18)$ & 12 & 171.3 & 0.85 & 0.93 & 0.73 & 0.17 & 0.12 & 0.22 & & 1.27 & 0.55 \\
\hline$(19,16)$ & 12 & 171.2 & 0.84 & 0.94 & 0.74 & 0.21 & 0.17 & 0.23 & 6.39 & 1.27 & 0.74 \\
\hline$(16,19)$ & 12 & 171.4 & 0.87 & 0.94 & 0.74 & 0.21 & 0.17 & 0.23 & & 1.27 & 0.76 \\
\hline$(20,15)$ & 12 & 171.1 & 0.85 & 0.91 & 0.74 & 0.20 & 0.15 & 0.22 & 6.56 & 1.23 & 0.66 \\
\hline$(15,20)$ & 12 & 171.2 & 0.87 & 0.94 & 0.74 & 0.21 & 0.17 & 0.22 & & 1.27 & 0.78 \\
\hline$(19,17)$ & 12 & 171.3 & 0.87 & 0.94 & 0.73 & 0.21 & 0.18 & 0.23 & 6.37 & 1.28 & 0.78 \\
\hline$(17,19)$ & 12 & 171.4 & 0.83 & 0.92 & 0.74 & 0.21 & 0.18 & 0.23 & & 1.25 & 0.80 \\
\hline
\end{tabular}




\begin{tabular}{c|c|c|c|c|c|c|c|c|c|c|c}
\hline 1 & 2 & 3 & 4 & 5 & 6 & 7 & 8 & 9 & 10 & 11 & 12 \\
\hline$(16,20)$ & 12 & 171.2 & 0.86 & 0.93 & 0.74 & 0.21 & 0.18 & 0.23 & & 1.25 & 0.78 \\
\hline$(20,16)$ & 12 & 171.1 & 0.86 & 0.92 & 0.74 & 0.20 & 0.18 & 0.23 & 6.36 & 1.25 & 0.78 \\
\hline$(19,18)$ & 12 & 171.3 & 0.84 & 0.92 & 0.73 & 0.17 & 0.11 & 0.22 & 6.37 & 1.26 & 0.49 \\
\hline$(18,19)$ & 12 & 171.4 & 0.87 & 0.94 & 0.74 & 0.17 & 0.13 & 0.22 & & 1.27 & 0.58 \\
\hline$(20,17)$ & 12 & 171.3 & 0.86 & 0.92 & 0.74 & 0.20 & 0.13 & 0.23 & 6.37 & 1.25 & 0.58 \\
\hline$(17,20)$ & 12 & 171.3 & 0.85 & 0.93 & 0.74 & 0.21 & 0.18 & 0.23 & & 1.27 & 0.80 \\
\hline$(18,20)$ & 13 & 171.3 & 0.82 & 0.93 & 0.73 & 0.22 & 0.22 & 0.22 & & 1.26 & 1.00 \\
\hline$(20,18)$ & 13 & 171.2 & 0.84 & 0.92 & 0.73 & 0.21 & 0.18 & 0.23 & 6.51 & 1.26 & 0.80 \\
\hline$(19,20)$ & 13 & 171.3 & 0.85 & 0.90 & 0.73 & 0.16 & 0.09 & 0.21 & & 1.23 & 0.40 \\
\hline$(20,19)$ & 13 & 171.3 & 0.83 & 0.92 & 0.73 & 0.16 & 0.10 & 0.22 & 6.48 & 1.27 & 0.45 \\
\hline
\end{tabular}

The torsional vibration frequency, $f$, was determined from the system cohesion energy changes, the other variables are: $L$ - sample length, $\rho$ - density. To obtain correct results, the initial sample torsion had to be large enough for the system energy change to be clearly larger than the changes resulting from thermal vibrations.

All the obtained results are listed in Table 1. Extensive discussion of the results and their comparison with available experimental and theoretical data will be presented in a forthcoming paper.

\section{References}

[1] R.H. Baughman, A.A. Zakhidov, W.A. de Heer, Carbon Nanotubes - the Route Toward Applications. Science 297 (5582), 787-792 (2002).

[2] Y.I. Prylutskyy, S.S. Durov, O.V. Ogloblya, E.V. Buzane- va, P. Scharff, Molecular dynamics simulation of mechanical, vibrational and electronic properties of carbon nanotubes. Computational Materials Science 17, 352-355 (2000).

[3] H.J. Qi, K.B.K. Teo, K.K.S. Lau, M.C. Boyce, W.I. Milne, J. Robertson, K.K. Gleason, Determination of mechanical properties of carbon nanotubes and vertically aligned carbon nanotube forests using nanoindentation. Journal of the Mechanics and Physics of Solids 51, 2213-2237 (2003).

[4] A. Krishnan, E. Dujardin, T.W. Ebbesen, P.N. Yianilos, M.M.J. Treacy, Young's modulus of single-walled nanotubes. Physical Review B 58, 1413-1419 (1998).

[5] S.J. Stuart, A.B. Tutein, J.A. Harrison, A reactive potential for hydrocarbons with intermolecular interactions. Journal of Chemical Physics 112, 6472-6486 (2000).

[6] S. Nosé, A molecular dynamics method for simulations in the canonical ensemble. Molecular Physics 52, 255-268 (1984).

[7] M. Bialoskorski, J. Rybicki, Mechanical properties of the carbon nanotubes: simulation program and exemplary results. Proc. of the $8^{\text {th }}$ Workshop of PTSK GdańskSobieszewo, p. 8 (2001).

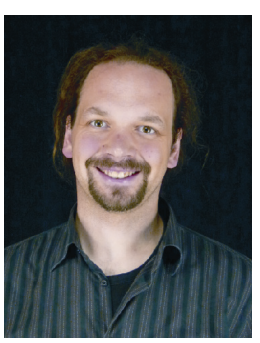

Michal BialoskórSKI was born in 1977 in Gdynia, Poland. In 2001 he received his M.Sc. degree and in 2011 his PhD degree in Physics at the Gdansk University of Technology. Presently, his field of interest include mechanical properties of nanostructures and computational simulations of nanomechanical systems.

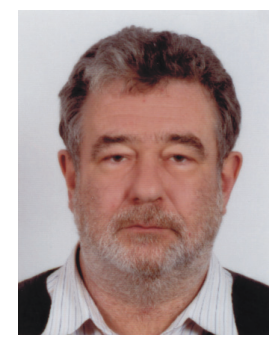

JAROSLAW RYBICKI is a professor in theoretical and computational physics at the Faculty of Applied Physics and Mathematics of the Gdansk University of Technology, Gdansk, Poland. His field of interest covers structure of disordered systems (oxide glasses and liquid metals and alloys), phase transitions (condensation from gas phase, premelting phenomena), mechanical properties of nanostructures (mechanisms of plastic deformation, formation and motion of dislocations, molecular mechanisms of friction). Research tools: classical and quatnum-classical simulations with particles, stochastic geometry methods. 
